\title{
Architecture Design of H.264/AVC Decoder with Hybrid Task Pipelining for High Definition Videos
}

\author{
To-Wei Chen, Yu-Wen Huang, Tung-Chien Chen, Yu-Han Chen, Chuan-Yung Tsai and Liang-Gee Chen \\ DSP/IC Design Lab, Graduate Institute of Electronics Engineering and Department of Electrical Engineering \\ National Taiwan University, Taipei, Taiwan; Email: towei@ video.ee.ntu.edu.tw
}

\begin{abstract}
The most critical issue of an H.264/AVC decoder is the system architecture design with balanced pipelining schedules and proper degrees of parallelism. In this paper, a hybrid task pipelining scheme is first presented to greatly reduce the internal memory size and bandwidth. Block-level, macroblocklevel, and macroblock/frame-level pipelining schedules are arranged for CAVLD/IQ/IT/INTRA_PRED, INTER_PRED, and DEBLOCK, respectively. Appropriate degrees of parallelism for each pipeline task are also proposed. Moreover, efficient modules are contributed. The CAVLD unit smoothly decodes bitstream into symbols without bubble cycles. The INTER_PRED unit highly exploits the data reuse between interpolation windows of neighboring blocks to save $60 \%$ of external memory bandwidth. DEBLOCK unit doubles the processing capability of our previous work with only $35.3 \%$ of logic gate count overhead. The proposed baseline profile decoder architecture can support up to $2048 \times 102430$ fps videos with $217 \mathrm{~K}$ logic gates, 10KB SRAMs, and $528.9 \mathrm{MB} / \mathrm{s}$ bus bandwidth when operating at $120 \mathrm{MHz}$.
\end{abstract}

\section{INTRODUCTION}

ISO/IEC Moving Picture Experts Group (MPEG) and ITUT Video Coding Experts Group (VCEG) formed a Joint Video Team (JVT) to develop the latest video coding standard, H.264/AVC [1]. It can save $25 \%-45 \%$ and $50 \%-75 \%$ of bitrates when compared with MPEG-4 ASP and MPEG-2, respectively. The bitrate saving in baseline profile comes from the new features including multi-frame and variable-blocksize (VBS) inter prediction, intra prediction, context-based adaptive variable length decoding (CAVLD), and deblock filter. However, an H.264/AVC decoder requires two times computational power of an MPEG-4 decoder, and four times of an MPEG-2 decoder. Therefore, hardware acceleration for H.264/AVC decoding is a must.

In this paper, there are four critical issues to be addressed. First, the decoding procedures of H.264/AVC are significantly more complex than those in previous standards, making the conventional macroblock (MB) pipelining very inefficient. Second, the context formation of CAVLD is highly dependent of prior symbols, bringing tough challenges to parallel processing. Third, the inter prediction with VBS and quarterpel precision is extrememly hungry for off-chip memory bandwidth, leading to the system throughput bottleneck. Fourth, the 2-D deblock filter fetches and processes each pixel for many times, requiring a huge amount of on-chip memory bandwidth and a high degree of computing parallelism.

The rest of this paper is organized as follows. In Section II, a hybrid task pipelining scheme is presented to reduce the internal memory size and to highly reuse the external memory data. Besides, the degrees of parallelism are analyzed. Section III describes three important modules in H.264/AVC decoders. Implementation results are shown in Section IV. Finally, Section V gives a conclusion.

\section{System ARCHITECTURE}

\section{A. Hybrid Task Pipelining Architecture}

The overall system architecture is shown in Fig. 1. According to our software/hardware partition scheme, the sequence parameter set (SPS), picutre parameter set (PPS), and slice headers are parsed by RISC because their symbol rates are very low. The MB-level information including MB headers and prediction residues are decoded by the PARSER Engine. The predicted pixels are generated by the INTER_PRED or INTRA_PRED Engine according to the MB type. The residues are generated by the IQ/IT Engine. The MB is reconstructed by SUM_AND_CLIPPING module. Finally, DEBLOCK Engine filters $\mathrm{MB}$ pixels and outputs them to the external memory. The buffers between the processing engines are required to separate pipelining stages. In summary, the system architecture is based on a hybrid task pipelining scheme including $4 \times 4$-block-level pipelining, MB-level pipelining, and frame-level pipelining. Previous designs of video decoders are usually based on MB pipeline scheme [2]. In H.264, $4 \times 4$-block is the smallest element of the prediction block type. The transforms and entropy coding are also based on $4 \times 4$-blocks. Based on these characteristics, a $4 \times 4$-block pipelining scheme for CAVLD, inverse quantization/inverse transformation (IQ/IT), and intra prediction (INTRA_PRED) is proposed with the benefit of less coding latency. It requires only $1 / 24$ of buffer size when compared to the traditional MB pipeline architecture.

Figure 2 explains the proposed $4 \times 4$-block pipeline scheduling. Each box labeled with a number represents a $4 \times 4$-block, the horizontal axis represents time, and the boxes of the same time slot denote concurrent pipeline tasks. Following the decoding order, the decoder processes one luma DC block (if existed), 16 luma blocks, two chroma DC blocks, 4 chroma (u) blocks, and finally 4 chroma (v) blocks.

Inter prediction produces the predicted MB pixels from previously decoded reference frames. In H.264, a number of different block sizes can be used for inter prediction$16 \times 16,16 \times 8,8 \times 16,8 \times 8,8 \times 4,4 \times 8$, and $4 \times 4$. As with intra prediction, the basic processing element of INTER_PRED is also a $4 \times 4$-block. As shown in Fig. 3(a), due to the sixtap FIR filter for interpolation, $9 \times 9$ interger reference pixels 


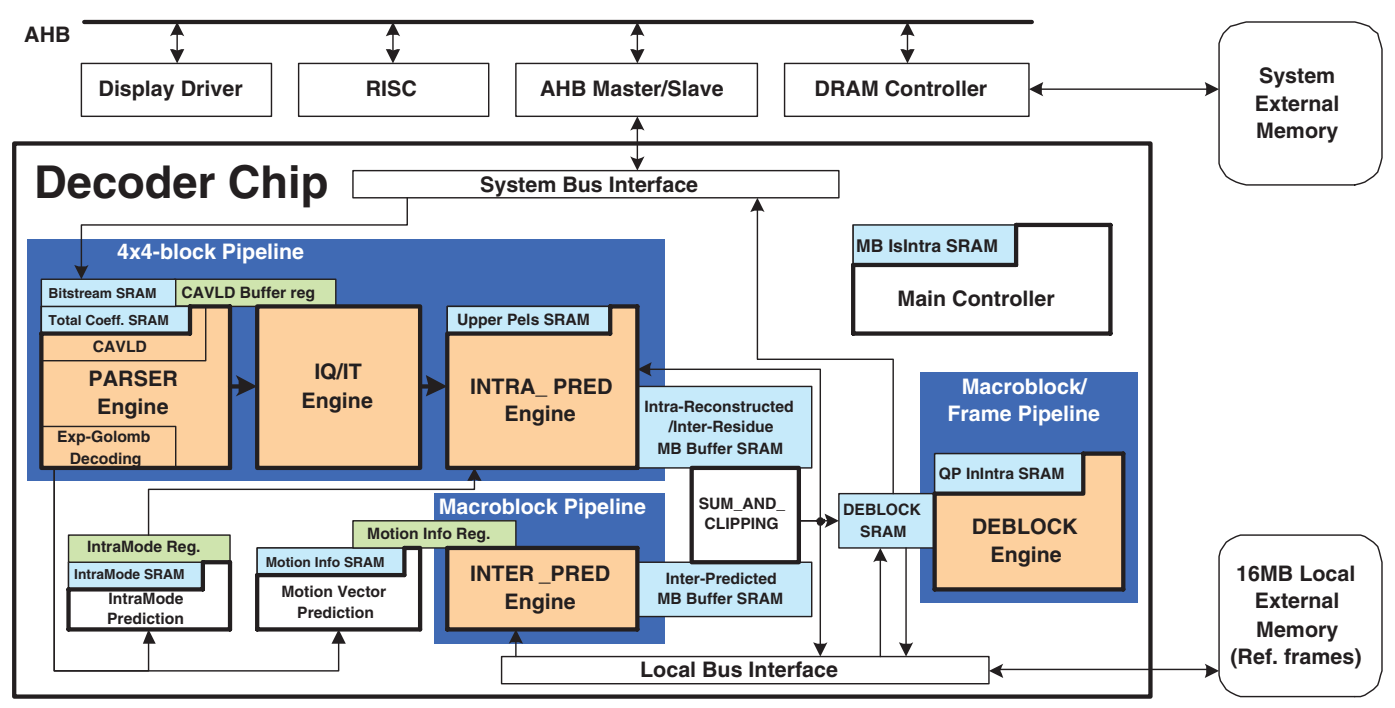

Fig. 1. Proposed system architecture.

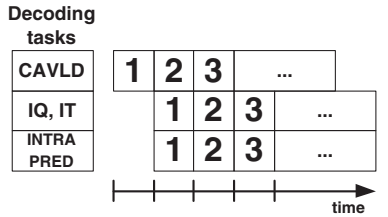

Fig. 2. Proposed $4 \times 4$-block pipeline scheme. Each box represents a $4 \times 4$ block. The number on each $4 \times 4$-block is the $4 \times 4$-block index.

are required for a current $4 \times 4$-block. If the block size is larger than $4 \times 4$ (in Fig. 3 (b), it is a $4 \times 8$-block), motion vectors of adjacent $4 \times 4$-blocks are the same. Overlapped reference frame pixels of these two $4 \times 4$-blocks can be shared and reused to reduce the bandwidth. It should be noted that the probability for large block size is considerably high [3]. The inherent order of $4 \times 4$-blocks in the bitstream is the double-z-scan order as illustrated in Fig. 4. Reference frame data reuse will be less efficient if INTER_PRED adopts the $4 \times 4$-block pipelining scheme and follows the double-z-scan order. For example, block 3 and block 9 in Fig. 4 cannot share reference frame data efficiently under the double-zscan order. Therefore, INTER_PRED should be scheduled to MB-level pipelining with a customized scan order to exploit the reference frame data reuse. All reference frame pixels necessary to inter predict a $\mathrm{MB}$ are read from memory in one pass to minimize memory bandwidth.

The basic processing element of H.264 deblock filtering is an MB. After all vertical edges of adjacent $4 \times 4$-blocks are filtered, the horizontal edges of adjacent $4 \times 4$-blocks are filtered. This data dependency makes $4 \times 4$-block pipeline for deblock filter impractical. If deblock operation has to be fit into the $4 \times 4$-block pipelining, an extra MB buffer is required because the block scanning order with efficient data reuse of deblock operation is completely different from that of previous stage. As a result, the deblock operation should be done in an MB-based schedule. When H.264 supports Flexible

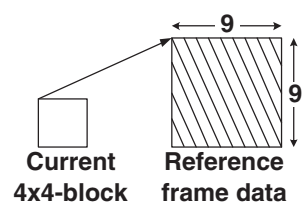

(a)

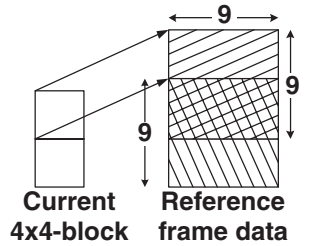

(b)
Fig. 3. (a) Inter prediction for a $4 \times 4$-block. (b) Inter prediction for one $4 \times 8$-block.

\begin{tabular}{|l|l|l|l|}
\hline 1 & 2 & 5 & 6 \\
\hline 3 & 4 & 7 & 6 \\
\hline 9 & 10 & 11 & 12 \\
\hline 15 & 14 & 15 & 16 \\
\hline
\end{tabular}

Fig. 4. Double-z-scan order of $4 \times 4$-blocks. The number and the arrow represent the $4 \times 4$-block order.

Macroblock Ordering (FMO) and Arbitrary Slice Ordering (ASO), the receiving order of MBs is not raster-scan order anymore, which violates of the scan order of the deblock operation. In the case of FMO and ASO, deblock filter should be scheduled to the frame-level pipelining. If FMO and ASO are not activated, the MBs are still received in raster-scan order, and the MB pipelining scheme can still be adopted.

The pipeline scheduling of the entire decoder is shown in Fig. 5 . MB 1, 2, and 3 are assumed to be inter predicted, while MB 4 is assumed to be an intra predicted MB. When inter predicted type MBs are processed, the intra prediction unit in the $4 \times 4$-block pipeline is disabled. On the other hand, when intra predicted type MBs are processed, the inter prediction unit in the MB pipeline is disabled.

\section{B. Parallelism Analysis}

Of the three $4 \times 4$-block pipeline tasks, CAVLD decodes unfixed number of symbols and takes unfixed cycles, while the 


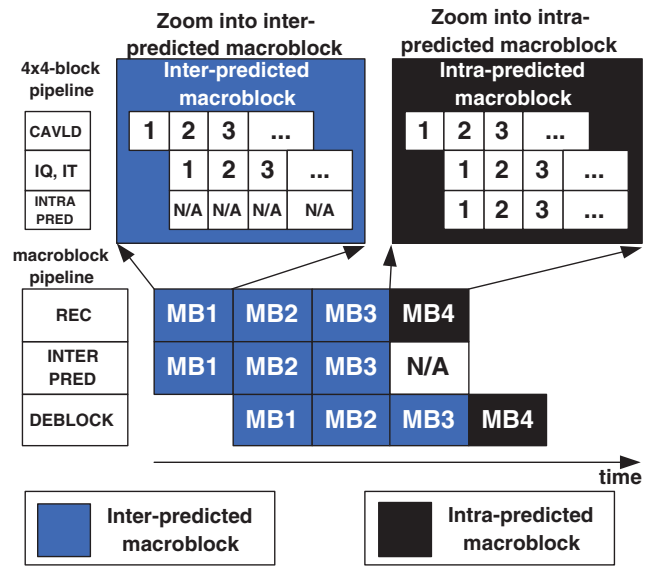

Fig. 5. Block-level/MB-level pipeline scheduling. N/A means the task is disabled and not available.

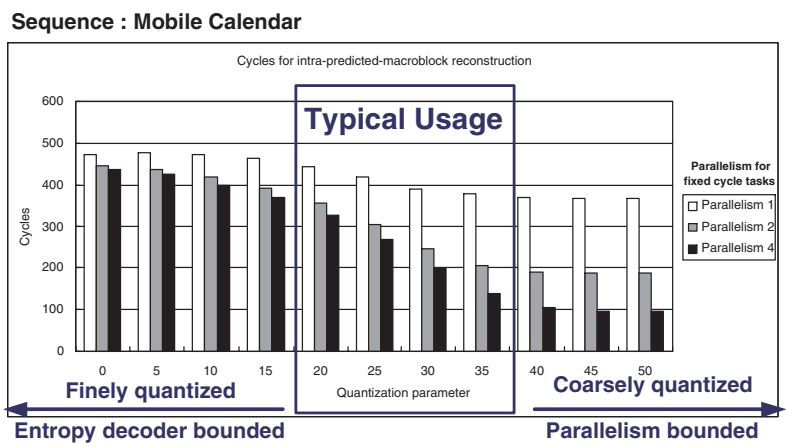

Fig. 6. The cycle count for decoding one intra MB.

other two tasks, IQ/IT and INTRA_PRED, take fixed cycles. Assuming CAVLD decodes one symbol per clock cycle, the degrees of parallelism of IQ/IT and INTRA_PRED remain to be determined. Here we discuss the degrees of parallelism in terms of module throughput. Different degrees of parallelism lead to different cycle time to complete the task. The simulated cycles to reconstruct an intra type MB for $4 \times 4$-block pipelining architecture is shown in Fig. 6. Finely quantized video results in more coefficients for CAVLD, and the total cycle time remains long even if the parallelism of fixed cycle tasks (IQ/IT and INTRA_PRED) is increased. In this case, the pipelining system performance is "CAVLD bounded". On the contrary, coarsely quantized video results in complementary phenomenon. In this case, the system is bounded by the parallelism of fixed cycle tasks. It should be noted that in our module designs, an $M$-parallel architecture has $M$ times of throughput, while the induced area overhead is much less than $M$ times [4]. To support $2048 \times 102430 \mathrm{fps}$ videos, the degrees of parallelism for IQ/IT and INTRA_PRED are chosen as 4. INTER_PRED produces the same number of predicted pixels as INTRA_PRED, so the parallelism of INTER_PRED is chosen the same as INTRA_PRED.

\section{Module Architecture}

In this section, we contributed three innovative modulesCAVLD, INTER_PRED, and DEBLOCK. For architecture of

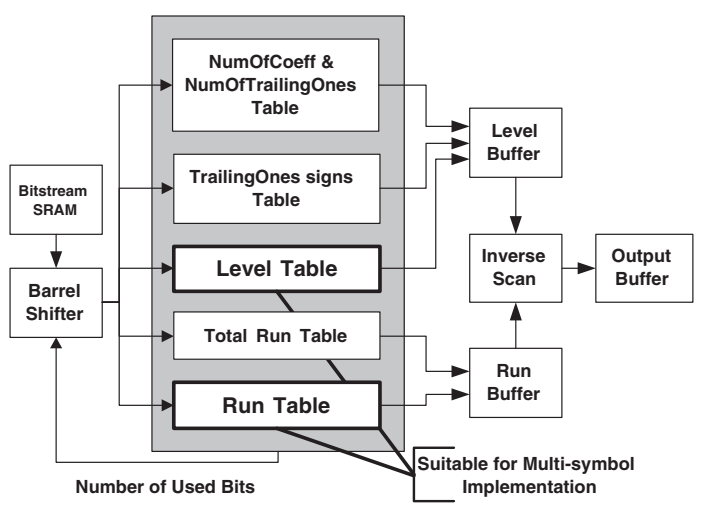

Fig. 7. Hardware architecture for CAVLD.

TABLE I

Summary OF Single-Symbol AND MULTI-Symbol CAVLD ENGINE

\begin{tabular}{lccc}
\hline & Single-symbol & Two-symbol & Three-symbol \\
\hline \hline Area & $100 \%$ & $120 \%$ & $140 \%$ \\
Cycles Per MB & 471 & 276 & 212 \\
\hline
\end{tabular}

The quantization parameter is set to 0 to demonstrate the capability of different architecture.

IQ/IT and INTRA_PRED module, please refer to the previous work [4].

\section{A. CAVLD}

The hardware architecture of CAVLD is shown in Fig.7. Each symbol can be consecutively decoded without bubble cycles. The intuitive parallel processing techniques to speed up CAVLD cannot be applied due to the context-based adaptive nature. In some ultra-high-end or low-power applications, the cycle time available to decode an MB is very limited. In these cases, single-symbol decoding engine for CAVLD appears as the throughput bottleneck of the system. Thus we proposed a multi-symbol decoding engine for CAVLD to overcome this limit. If there are $N$ possible symbols to be decoded, for a two-symbol decoding engine, there will be $N^{2}$ possible jointsymbols, which will be too complicated. After analysis of the CAVLD algorithm, it shows that multi-symbol decoding engine can be much more simplified. There are only two kinds of symbol with high possibility to occur consecutively in bitstream, which are levels and runs. Building multi-symbol VLD tables for levels and runs, CAVLD can decode consecutive multiple levels or runs in one cycle. Simulation results show that cycle count for CAVLD can be greatly reduced with low area overhead, as shown in Table I.

\section{B. Inter Prediction}

In H.264, VBS inter prediction are composed of $4 \times 4$ blocks. Based on this characteristic, we can design a $4 \times 4$ block inter prediction unit, and reuse it for larger block type. The inter prediction process involves six-tap filtering, so $9 \times 9$ reference pixels must be loaded for a $4 \times 4$-block. As a result, inter prediction demands for large bandwidth and becomes the system bottleneck. Two techniques are proposed to deal with 
TABLE II

Chip SPECIFICATION

\begin{tabular}{lcccc}
\hline & Proposed & C\&S [2] & Conexant [5] & SandVideo [6] \\
\hline \hline Technology & $180 \mathrm{~nm}$ & $130 \mathrm{~nm}$ & $130 \mathrm{~nm}$ & $130 \mathrm{~nm}$ \\
Logic Gates & $217 \mathrm{~K}$ & $910 \mathrm{~K}$ & $300 \mathrm{~K}$ & N/A \\
Memory (Internal/External) & $10 \mathrm{~KB} / 16 \mathrm{MB}$ & N/A & $74 \mathrm{~KB} / 24 \mathrm{MB}$ & N/A \\
Operating Frequency & $120 \mathrm{MHz}$ & $170 \mathrm{MHz}$ & $200 \mathrm{MHz}$ & N/A \\
Profile & baseline & baseline(multi-standards) & main & main \\
Spec. & $2048 \times 1024,30 \mathrm{fps}$ & $1920 \times 1080,30 \mathrm{fps}$ & $2048 \times 1024,30 \mathrm{fps}$ & $2048 \times 1024,30 \mathrm{fps}$ \\
\hline
\end{tabular}

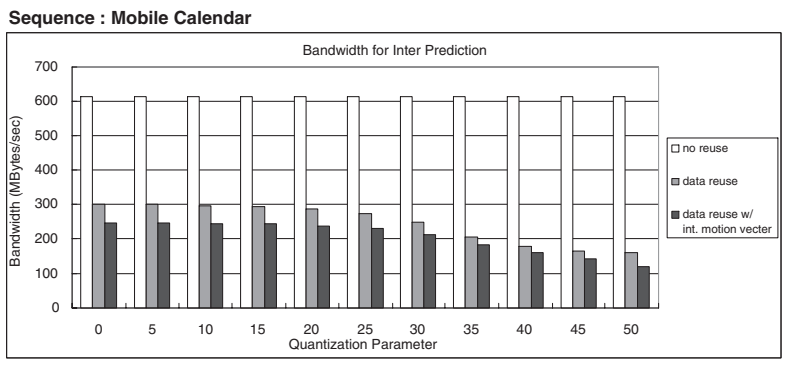

Fig. 8. Bandwidth for inter prediction under different reference frame reuse scheme.

this bandwidth problem. First, reference frame pixels shared by adjacent $4 \times 4$-blocks should be reused. Second, if motion vectors point to integer-pixel positions in reference frame, interpolation is not necessary, and less pixels are required, therefore further bandwidth reduction can be achieved. Simulation results in Fig. 8 show that bandwidth can be reduced up to $60 \%$ using these two techniques.

\section{Deblock Filter}

In our previous work [7], we proposed the architecture for an H.264 deblock filter. A parallel-in parallel-out FIR filter and an array of $8 \times 48$-bit shift registers with reconfigurable data path is implemented to support both horizontal filtering and vertical filtering on the same circuit. In this paper, in order to support high-end levels defined in the standard, a new architecture for deblock filter, shown in Fig. 9, with double throughput is proposed. While the original memory allocation scheme uses only one dual-port SRAM, the proposed doublethroughput scheme splits from one dual-port SRAM to two half-sized dual-port SRAMs. By this new architecture, only one additional filter logic is required to double the throughput. The transpose buffer, all information registers, boundarystrength calculating logic remained the same. The induced gate count overhead is only $35.3 \%$ compared to our previous work.

\section{IMPLEMENTATION RESULTS}

We described our architecture by Verilog HDL and synthesized the circuit using 180nm Artisan CMOS cell library by Synopsys Design Analyzer with critical path constraint set to $8.33 \mathrm{~ns}(120 \mathrm{MHz})$. The results are shown and compared with other implementations in Table II. Our work has the benefit of lower gate count, lower internal/external memory requirements, and lower operating frequency. Currently, we

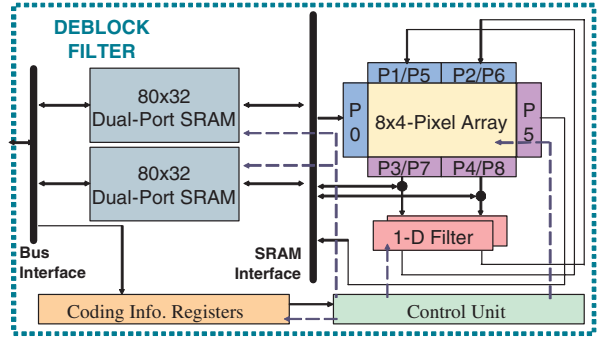

Fig. 9. Proposed new deblock filter architecture.

only support the baseline profile. In the future, our technology will be improved to $130 \mathrm{~nm}$, and the functionality will be extended to the main profile.

\section{CONCLUSION}

In this paper, we contributed a hardware architecture for H.264/AVC decoders. The major idea is to arrange decoding functions into proper pipelining schedules. Degrees of parallelism are also chosen to meet real-time requirements. The external memory bandwidth is greatly reduced due to efficient data reuse scheme. Simulation results show that the processing capability of proposed architecture is to support $2048 \times 1024$ 30fps videos at $120 \mathrm{MHz}$, and the silicon area and required frequency are lower than other commercial H.264 decoders.

\section{REFERENCES}

[1] J. V. Team, Draft ITU-T Recommendation and Final Draft International Standard of Joint Video Specification. ITU-T Rec. H.264 and ISO/IEC 14496-10 AVC, May 2003.

[2] H. Y. Kang, K. A. Jeong, J. Y. Bae, Y. S. Lee, and S. H. Lee, "MPEG4 AVC/H.264 decoder with scalable bus architecture and dual memory controller," in Proc. of Int. Symposium on Circuits and Systems (ISCAS'04), 2004.

[3] Y. W. Huang, B. Y. Hsieh, T. C. Wang, S. Y. Chien, S. Y. Ma, C. F. Shen, , and L. G. Chen, "Analysis and reduction of reference frames for motion estimation in MPEG-4 AVC/JVT/H.264," in Proc. of Int. Conf. on Accoustics, Speech, and Signal Processing (ICASSP'03), 2003, pp. III - 145-8 vol.3.

[4] Y. W. Huang, B. Y. Hsieh, T. C. Chen, and L. G. Chen, "Hardware architecture design for H.264/AVC intra frame coder," in Proc. of Int. Symposium on Circuits and Systems (ISCAS'04), 2004.

[5] Y. Hu, A. Simpson, K. McAdoo, and J. Cush, "A high definition H.264/AVC hardware video decoder core for multimedia SoC's," in Proc. of Int. Symposium on Consumer Electronics (ISCE'04), 2004.

[6] SandVideo, "Sandvideo releases the first h.264 decoder," in http://www.prweb.com/releases/2003/12/prweb93434.htm, 2003.

[7] Y. W. Huang, T. W. Chen, B. Y. Hsieh, T. C. Wang, T. H. Chang, and L. G. Chen, "Architecture design for deblocking filter in H.264/JVT/AVC," in Proc. of Int. Conf. Mutimedia and Expo (ICME'03), 2003, pp. I - 693-6 vol.1. 